\title{
Congrès des oncogènes, Frederick (juillet 1989) : phosphorylations, cycle cellulaire et oncogènes
}

Outre le résultat essentiel concernant la farnésylation des protéines p21 ris (voir nouvelle: cholestérol, oncogènes et cancer, page 600 de ce numéro), plusieurs autres résultats significatifs ont été présentés à Frederick, dans le Maryland, à côté de Bethesda (USA).

Une session complète a été consacrée aux phosphorylations au cours du cycle cellulaire, dans lesquelles la sérine-thréonine kinase $\mathrm{cdc}_{2}$ est impliquée. Cette kinase est probablement responsable de la phosphorylation du produit du gène $R b$ (gène de susceptibilité au rétinoblastome). Il semble établi que la protéine pl05 $5^{\mathrm{Rb}}$ est active à l'état déphosphorylé, et est alors capable de se fixer sur les oncogènes nucléaires $E_{1} A$ ou antigène $\mathrm{T}$ de $\mathrm{SV}-40$, et est inactive à l'état phosphorylé $\left(m / s n^{o} 4, v o l .5\right.$, p. 259). A l'état actif, non phosphorylé, pl05 ${ }^{\mathrm{Rb}}$ s'oppose au déclenchement de la mitose. Le groupe de J. Wang (UCSD, La Jolla, CA, USA) a démontré que les sites de phosphorylation de $\mathrm{pl} 05^{\mathrm{Rb}}$ par $\mathrm{cdc}_{2}$ in vitro correspondaient exactement aux sites de phosphorylation de cette protéine in vivo. $\mathrm{Cdc}_{2}$ est donc probablement la kinase responsable de l'inactivation du produit de l'anti-oncogène $R b$. Par ailleurs, les équipes de D. Beach (Cold Spring Harbor, NY, USA) et J. Wang ont montré que, en phase $S$ et $G_{2}$, cdc $c_{2}$ était phosphorylée sur des tyrosines, puis complètement déphosphorylée lors de l'entrée en mitose. C'est la forme déphosphorylée de $\mathrm{cdc}_{2}$ dans des cellules en métaphase qui a l'activité histonekinase la plus élevée. Des inhibiteurs de tyrosine phosphatases qui empêchent la déphosphorylation des tyrosines phosphorylées (comme le vana- date) bloquent les cellules en phase $\mathrm{G}_{2}$ et empêchent l'entrée en mitose (voir aussi l'article de Christian Le Peuch, sous presse dans médecinel sciences)

Les équipes de D. Shalloway (Penn State University, PA, USA), de D. Bishop (UCSF, CA, USA) et de $\mathrm{H}$. Varmus (UCSF, CA, USA) ont montré que la kinase p60 $0^{\text {src }}$ était un substrat de $\mathrm{cdc}_{2}$ et était phosphorylée par cette enzyme sur deux résidus thréonine (résidus 34 et 46 ) et une sérine (résidu 72), au cours de la mitose. Ainsi, les effets de $\mathrm{cdc}_{2}$ sur le cycle cellulaire passent également par le produit de l'oncogène $c$-src qui, en retour, phosphoryle $\mathrm{cdc}_{2}$ sur des résidus tyrosine.

M. Resh (Princeton, University, NJ, USA) a démontré que l'ancrage de p60 src à la membrane, par l'intermédiaire d'un myristate lié à la glycine $\mathrm{N}$-terminale, nécessitait une interaction avec une protéine membranaire. Il s'agit en fait d'un véritable récepteur pour le myristate, qui doit reconnaître, outre le myristate, les premiers résidus (une douzaine) de la chaîne protéique. Un peptide myristilé comportant les douze premiers résidus de $\mathrm{p} 60^{\mathrm{v}-\mathrm{src}}$ inhibe la liaison à la membrane de $\mathrm{p} 60^{\mathrm{v}-\mathrm{src}}$ myristilés. En revanche, le même peptide non myristilé, ou bien un peptide myristilé correspondant à une autre protéine de la famille src (protéines qui sont toutes différentes en $\mathrm{N}$-terminal), n'inhibe pas la liaison de p60 v-src myristilé à la membrane. Il y aurait donc un récepteur différent pour chaque protéine de la famille src (huit sont décrites à ce jour).

L'équipe de B. Sefton (Salk Institute, San Diego, CA, USA) a cartographié la zone d'interaction CD4/p56 $6^{\text {lck }}$ (la p56 $6^{\text {lck }}$ est une tyrosine kinase des lymphocytes T) (voir l'article de Rémy Fagard, p.554 de ce numéro), respectivement sur la partie C-terminale de CD4 et $\mathrm{N}$-terminale de p56 $6^{\text {lck. }}$. Par ailleurs, l'activation de la protéine kinase $\mathrm{C}$ par les esters de phorbol dissocie le complexe CD4/p56 $6^{\text {lck }}$, mais non le complexe CD8/p56 $6^{\text {lck }}$, ce qui suggère que la régulation de la p56 $6^{\text {lck }}$ par ces deux molécules est différente. Enfin, cette équipe a trouvé une corrélation entre la perte d'activité tyrosine phosphatase, dans certaines lignées lymphocytaires déficientes en CD45, et l'hyperphosphorylation de la p56 $6^{\text {lck }}$ au site tyrosine 505 C-terminal, ce qui contrôlerait négativement l'activité kinasique de cette enzyme. Ainsi, la phosphatase CD45 jouerait un rôle dans la déphosphorylation de ce résidu.

L'équipe de C. Sherr (St Jude Hospital, Memphis, TE, USA) a démontré que l'activation de la protéine kinase $\mathrm{C}$ dans des macrophages induisait une modulation négative du récepteur du CSF-1 (colony stimulating factor 1 , spécifique des monocytes/macrophages), par survenue d'un clivage protéolytique du domaine extracellulaire de liaison du récepteur.

P. Sutrave et S.H. Hughes (NCI, Frederick, MD, USA) ont obtenu des souris transgéniques avec le protooncogène $c$-ski, placé sous le contrôle du LTR du virus du sarcome murin. L'hyperexpression de $c$-ski chez les souris se traduit par une hypertrophie importante des masses musculaires par rapport aux souris normales: c'est le body-building par transgénèse !

R. B. 